\title{
Nurse-Driven Training Courses: Impact on Implementation of Ambulatory Blood Pressure Monitoring
}

\author{
Estel Félez-Carrobé ${ }^{*}, 1,2$, Maria Sagarra-Tió ${ }^{1}$, Araceli Romero ${ }^{1}$, Montserrat Rubio ${ }^{1}$, Lourdes Planas ${ }^{1}$, \\ María José Pérez-Lucena ${ }^{3}$, Montserrat Baiget ${ }^{4}$, Cristina Cabistañ ${ }^{1}$ and Jordi Félez ${ }^{1}$ \\ ${ }^{1} A B S$ Canaletes, ${ }^{2} A B S$ Guinardó and ${ }^{3} A B S$ Serraparera, Institut Català de la Salut. Cerdanyola del Vallès and \\ Barcelona, ${ }^{4}$ Genetics Department, Hospital Sant Pau i Santa Creu, Barcelona, Spain
}

\begin{abstract}
Background: Ambulatory blood pressure monitoring (ABPM) predicts cardiovascular risk and identifies whitecoat and masked hypertension, efficacy of treatment and the circadian cycle of hypertensive patients.

Objective: To analyze the effectiveness of ABPM implementation thoughtout a nurse-driven training program.

Materials and Methodology: Twenty eight professionals were involved in the study carried out in the primary care center of the metropolitan area of Barcelona that serves 34,289 inhabitants. The ABPM implementation program was driven by two nurses that held four education sessions. After a 2-year follow-up period, we assessed the outcome of attendance at the educational sessions. First, we evaluated whether the program increased the number of orders of ABPM. Second, we used a survey to evaluate to what extent the input of our educational sessions was understood by attendants. Third, we analyzed the effect ABPM results had on the treatment of patients with a bad control of their hypertension.
\end{abstract}

Results: After the training sessions we found a 6-fold increase in the number of patients undergoing ABPM. We analyzed 204 hypertensive individuals: $41 \%$ dippers, $34 \%$ were non-dippers, $20 \%$ were risers and 5\% were extremely dippers. According to our survey, $100 \%$ of attendants had a good practice regarding ABPM management. However only $27 \%$ of riser patients were studied with a second ABPM.

Conclusions: Specific training processes are needed for implementation of ABPM and an even more concentrated effort should be focused on training in the correct interpretation of ABPM results.

Keywords: Ambulatory, blood pressure, cardiovascular morbidity, educational program, essential hypertension, primary care.

\section{INTRODUCTION}

Arterial blood pressure (BP) is a complex and wellregulated hemodynamic process that allows the sustained flow of blood cells within the vascular system and enables the appropriate interchange of oxygen and $\mathrm{CO}_{2}$ in tissues. In addition to diurnal changes, blood pressure can be modulated by a wide variety of conditions, including emotional and environmental circumstances [1-4]. It is noteworthy that with only a single method to evaluate $\mathrm{BP}$, the office BP measurement (OBPM), hypertension has been identified and treated for many years. Combining the use of a broader variety of methods, which are easy and reliable, such as home and ambulatory BP measurements, better control of hypertension has now been accomplished. Home BP measurement (HBPM) for example, is currently used by patients with essential hypertension $(\mathrm{EH})$ and the advantage of this approach to measure and control $\mathrm{EH}$ is well established [2-5]. As a complementary tool, ambulatory BP measurement (ABPM, the automatic measurement of

\footnotetext{
*Address correspondence to this author at the ABS Canaletes and ABS Guinardó, InstitutCatalà de la Salut. Cerdanyola del Vallès and Barcelona, Spain; Tel: ++34 626013 832; Fax: ++34 9358636 96;
} E-mail: estelfelez@gmail.com brachial blood pressure at fixed time intervals during a 24-48 hour period away from a medical setting) was first introduced in hospitals and its use was restricted to $\mathrm{EH}$ patients admitted to specialized medical centers [5-7]. Fortunately, at present its use has dramatically changed and ABPM is mainly utilized in primary settings where the majority of EH patients are treated [2-7].

The disadvantages of ABPM compared with OBPM or HBPM, such as cuff discomfort and disturbed sleep are overcome by their advantages [2]. A wide variety of studies have demonstrated that ABPM is more reproducible and a better predictor of organ damage and incidence of cardiovascular events compared with OBPM [3, 6]. In addition, ABPM identifies the white coat phenomenon (increased $\mathrm{BP}$ in a doctor's office in patients with normal ambulatory $\mathrm{BP}$ ) and discovers masked hypertension (normal doctor's office BP in patients with high ambulatory BP [5-7]. ABPM also allows better control of BP in pregnant women with preeclampsia or eclampsia risk, subjects with diabetes and metabolic syndrome [3, 8-10]. ABPM is indicated in the clinical management of hypertensive patients with resistance to treatment (defined as BP above goal despite the use of three agents of different classes in optimal doses, including a diuretic) $[2,3]$. 
In addition, ABPM discriminates among patients with a dipping or with a non-dipping BP pattern and identifies patients with either a riser or extremely dipper pattern. Loss of the nocturnal dip has been associated with increased sympathetic tone and renal sodium reabsorption $[3,9,11$, 12]. Non-dippers are defined as patients who show a reduction in $\mathrm{BP}$ of less than $10 \%$ between average day and night BP determinations [3]. This status is associated with a higher prevalence of target organ damage, left ventricular hypertrophy, development of atherosclerotic plaques and intima-media thickness in women, higher urinary protein excretion and increased cardiovascular morbidity and mortality (reviewed in 3). The non-dipping pattern is also associated with diabetes and seen in patients with chronic renal failure [3, 12-14]. Risers and extremely dipper, (defined as patients who show an increase of more than $10 \%$ or more than a $20 \%$ decrease between average day and night BP, respectively), also have a higher cardiovascular risk [3, 14]. ABPM is the unique method to identify these distinct BP patterns and control changes in antihypertensive agents, or dose or timing of drugs, in order to improve BP control and reduce cardiovascular risk (CVR) [3, 12-14].

In light of the foregoing considerations, we carried out a nurse-driven ABPM training program in order to implement its use in a primary care center located in the metropolitan area of Barcelona (Spain). The program was evaluated after a 2-year follow-up period. The objective of this article is to discuss the evidence supporting the use of ABPM in primary care centers and the importance of specific training programs to implement the use of ABPM.

\section{MATERIALS AND METHODOLOGY}

Twenty-eight health professionals (14 nurses and 14 family doctors) participated in the study carried out during a two-year period. Four training sessions were conducted by 2 mentor nurses. In the first two sessions, methods to evaluate cardiovascular risk and the importance of good control over hypertension variables were stressed. The last two sessions were specifically focused on explaining the advantages of ABPM, such as the easy identification of white coat hypertension and obtaining information on the pattern of BP at rest (identification of a dipper, non- dipper, riser or extremely dipper pattern) and the clinical consequences of these specific patterns. In addition, information on how to modify treatment of hypertensive patients according to ABPM data was also discussed.

ABPM was carried out on two hundred and four individuals having at least one of the following characteristics: 1) a documented diagnosis of $\mathrm{EH} ; 2$ ) evaluated in a primary health care clinic with suspected $\mathrm{EH}$; and or 3) treated with antihypertensive drugs. In addition for all patients $>1$ month had elapsed between measurements of office BP and HBPM. Also, valid and reliable BP and HBPM information, complete data on all variables required for analysis of ABPM, as defined by the MAPAPRES protocol, was available for patients enrolled in the study [15]. The reasons for ordering an ABPM were stated on the order form and included six optional answers: 1) to evaluate efficacy of treatment; 2) to detect white coat syndrome; 3) to analyze high risk hypertension; 4) to define circadian rhythm; 5) to identify refractory hypertension; or 6) to observe labile hypertension.
Office BP was measured with validated semiautomatic devices, using appropriate cuffs ( 2 sizes), keeping the subject in a sitting position and ensuring standardized conditions [3, 16]. The average of 2 BP measurements was used for analyses. Thereafter, 24 hour ABPM was performed noninvasively on the non-dominant arm, using a Spacelab's Model 90207 device and spacing the readings at 20-minute or 30-minute intervals during the predicted periods of activity or rest, respectively. Patients were instructed to carry out their usual activities, keep their arm extended and immobile at the time of each cuff-inflation, and return the following day for device removal. ABPM was regarded as valid only if $>80 \%$ of systolic BPs (SBP) and diastolic BPs (DBP) during the active or rest time periods (from subject diaries) were satisfactory. All valid recordings were analyzed to obtain average 24-hour, active and at rest SBP and DBP. Nurses and physicians who carried out ABPM were trained and certified in ABPM.

Nurses and/or physicians fully completed a computerized order form for ABPM based upon interviews and physical examinations of patients at the time of visit and on data drawn from clinical records. The variables included, age, gender, clinical systolic and diastolic blood pressure respectively, duration of hypertension in years, antihypertensive drugs taken, time of drug taking, body mass index (BMI $\left.\left[\mathrm{g} / \mathrm{m}^{2}\right]\right)$, smoking habits, existence of dyslipidemia, diabetes, individual or family histories of premature cardiovascular disease, and target-organ damage. Dyslipidemia was defined as total serum cholesterol $>250 \mathrm{mg} / \mathrm{dL}$, low-density cholesterol $>155 \mathrm{mg} / \mathrm{dL}$ or high-density cholesterol $<40 \mathrm{mg} / \mathrm{dL}$ in men and $<48 \mathrm{mg} / \mathrm{dL}$ in women, respectively, or the presence of current lipid-lowering therapy; obesity as body mass index $>30 \mathrm{~kg} / \mathrm{m}^{2}$; diabetes mellitus as fasting blood glucose repeatedly $126 \mathrm{mg} / \mathrm{dL}$ or current antidiabetic therapy; microalbuminuria as average urinary albumin excretion of 30 to $300 \mathrm{mg}$ daily or albumin/ creatinine ratio $>22 \mathrm{mg} / \mathrm{g}$ in men and $>31 \mathrm{mg} / \mathrm{g}$ in women and proteinuria as urinary protein excretion $>300 \mathrm{mg}$ daily; left ventricular hypertrophy as left ventricular mass index calculated from a M-mode echocardiogram $>125 \mathrm{~g} / \mathrm{m}^{2}$ in or $>110 \mathrm{~g} / \mathrm{m}^{2}$ for women or the presence of electrocardiographic criteria (Sokoloff index $>35 \mathrm{~mm}$ ). Renal disease was diagnosed when serum creatinine was $>1.5 \mathrm{mg} / \mathrm{dL}$ in men and $>1.4$ $\mathrm{mg} / \mathrm{dL}$ in women or when proteinuria was present. Biochemical parameters from the last office-based determination within the preceding 3 months were used. The procedures followed were in accordance with institutional guidelines from Institut Català de la Salut (ICS) [17] and as mentioned, they were inclouded in the study in order to calculate ABPM results by MAPAPRES computerized protocol.

Clinical variables were compared using the $\chi^{2}$ test, as appropriate. Statistical significance was set at $P<0.05$. Average activity and resting-time ABPM was calculated as described following the protocol and guidelines of MAPAPRES [15].

\section{RESULTS}

Two hundred and four ABPM were carried out in our center. Gender distribution was equal: 103 males and 101 females with an average age of $53 \pm 14$ and $60 \pm 14$ year old for males and females, respectively. As mentioned, the 
reasons for ordering an ABPM study were stated on the order from. Most of the ABPM orders (38\% of patients) were done to determine whether HP patients were being treated correctly. The next most frequent reasons for ordering ABPM was to detect a white coat syndrome $(25 \%$ of subjects) and to analyze ABPM in high-risk hypertension ( $16 \%$ of individuals). Other reasons were to study: circadian rhythm $(12 \%)$, refractory hypertension $(5 \%)$ and labile hypertension (4\%). To characterize individuals with suspected $\mathrm{EH}$, with respect to their circadian rhythms, the recorded pattern of ABPM measurements was defined as dipper, non-dipper, riser or extremely dipper according to the criteria describe above. In our set of 204 individuals, 41\%

A

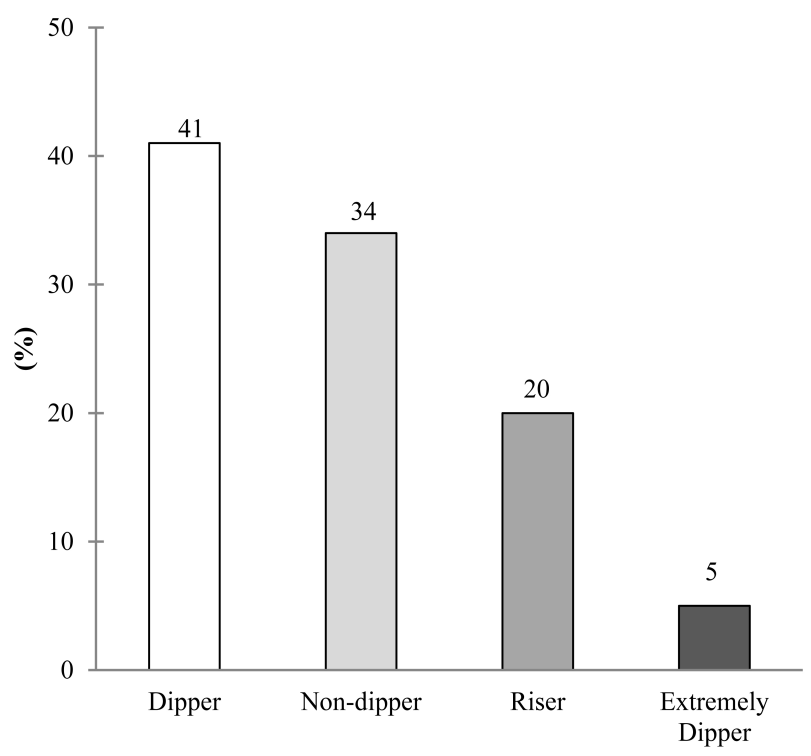

C

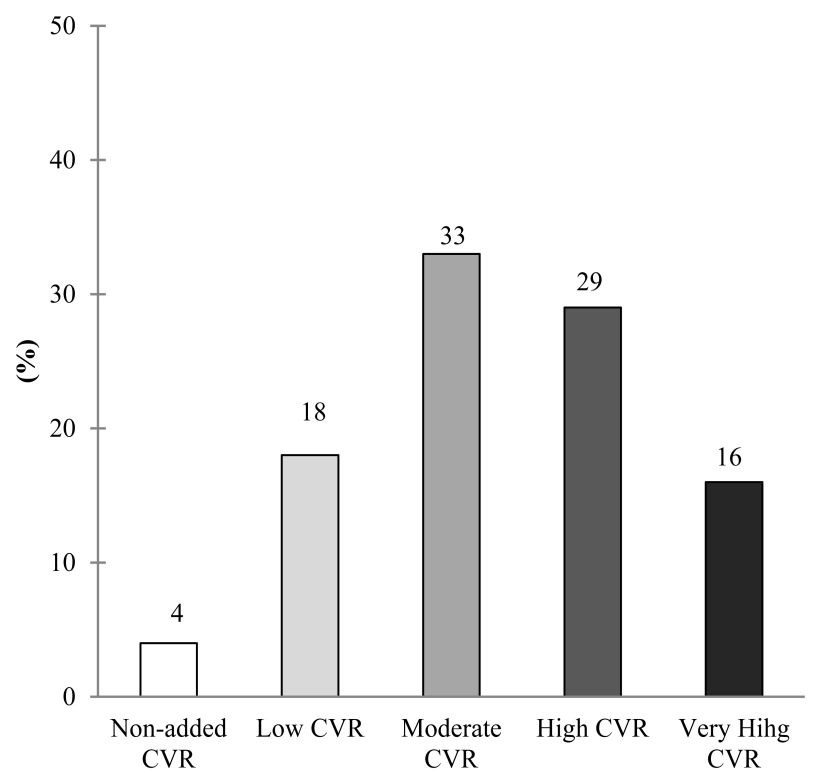

$(n=84)$ were dippers and $34 \%(n=69)$ were nondippers. Twenty $\%$ of subjects $(n=41)$ were risers and $5 \%(n=10)$ were extreme dippers. Fig. (1A) depicts these data as the percentage of circadian rhythm patterns analyzed. Summarizing these data, 80 out of 204 individuals had a dipper or extremely dipper pattern whereas 124 had a non-dipper or riser pattern.

According to ABPM results, $52 \%$ of individuals $(n=106)$ had BP levels under control. When subjects were divided by their circadian rhythm patterns, good BP control was observed in a similar percentage $(45.3 \pm 4.4 \%$, ranging from $51-40 \%$ ) among dipper, non dipper and extremely dipper

B

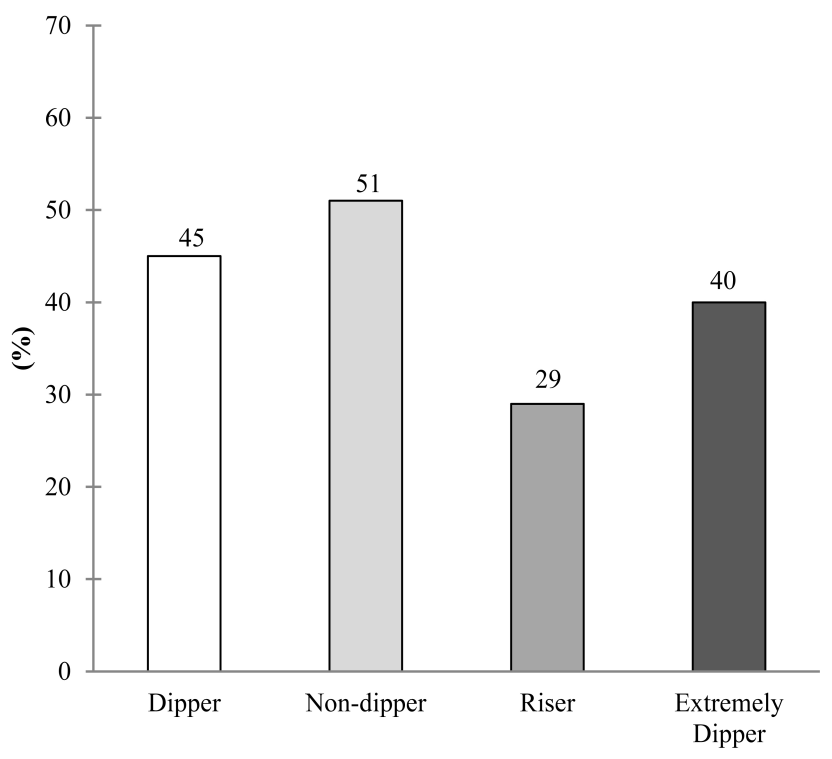

D

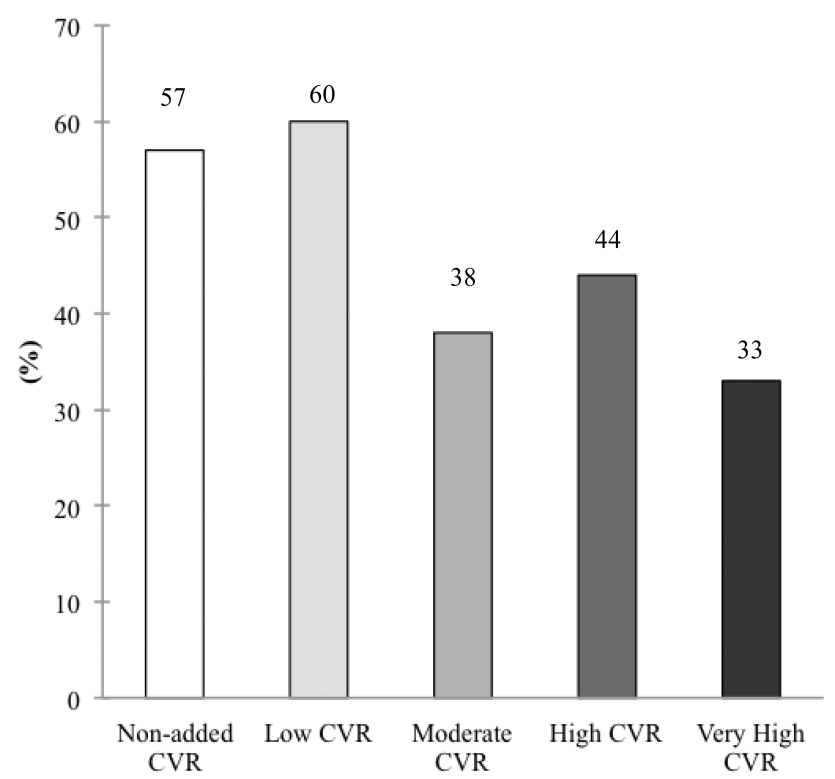

Fig. (1). Characterization of subjects included in the study. (A) Shows the percentage of individuals with a determined ABPM pattern as describe in the bar legends. The percentage of individuals with normal blood pressure values is expressed in (B) regarding their ABPM pattern. (C) Depicts the percentage of individuals with a distinct cardiovascular risk. (D) Shows the percentage of subjects with normal levels in their BP measurement concerning their cardiovascular risk. 
subjects while only $29 \%$ of riser individuals $(n=59)$ were under good BP control (Fig. 1B).

EH is associated with other CVR factors including age, gender, cholesterol levels (HDL and LDL), diabetes, smoking and body mass index and circadian rhythm type. Fig. (1C) summarize the percentage of subjects with a determined CVR among the population of individuals who underwent ABPM. CVR ranged from non-added to very high risk factors (see details in the legend to Fig. 1). Based on this categorization, the percentage of individuals with controlled BP levels as measured by ABPM is indicated in Fig. (1D).

Once we had obtained data from the population of subjects who had undergone ABPM, we wished to determine whether nurse driven educational intervention among health care professionals had any impact on ABPM management. That was explored using three different approaches. First, we evaluated the number of ABPM orders both before and after educational training. Before educational intervention we recorded 2.5 orders per month and this number increased to 8.9 and 15.5 orders per month following the first and second educational interventions, respectively. The health professionals in our primary care center were grouped in Health Basic Units (HBU) composed of a nurse and a family doctor. We had $14 \mathrm{HBU}$ and we recorded the attendance at the educational sessions for each HBU. Maximal attendance reflected the attendance of both the nurse and the doctor at the 2 sessions and scored 4 points while 0 points were scored when neither nurse nor doctor from a particular HBU attended any session. Consequently, we designated HBUs as attendant when a nurse or doctor from a particular HBU had attended 3 or more sessions or non-attendant when a nurse or doctor had attended less than 3 sessions.

Attendants, which represented $50 \%$ of the HBU, ordered 173 ABPM (85\%) whereas non-attendant HBUs that can be considered control group, ordered 31 ABPM (15\%). Fig. (2) depicts these data. A 3 -fold increase in ABPM orders was observed after the second educational intervention in attendant HBUs but it is noteworthy that even in nonattendant HBUs a marked relative increase in ABPM orders was observed (2.3-fold increase). The $\mathrm{p}$ values were also significant ( $p>0.05)$ comparing orders among attendants and non-attendants after the 1 st and 2nd educational specific ABPM sessions.

As a second approach to evaluate the efficiency of training in ABPM, a survey related to the educational interventions was carried out. The survey included data on professional identification and also questions such as: Why didn't you ever order an ABPM? Among three professionals in this situation the answers were: "did not know the program", "preferred home BP monitoring" and "no reason provided". In this survey we also checked whether the perception between then number of orders written and the orders actually done corresponded. Among attendant HBUs only one discrepancy was found between nurse and doctor, whereas four contradictions were found among the seven non-attendants HBU. Among 13 professionals who believed they did not order many ABPM, and in fact they did not, 3 believed it was much work, 2 believed it was uncomfortable for patients, 2 did not know how to manage results and

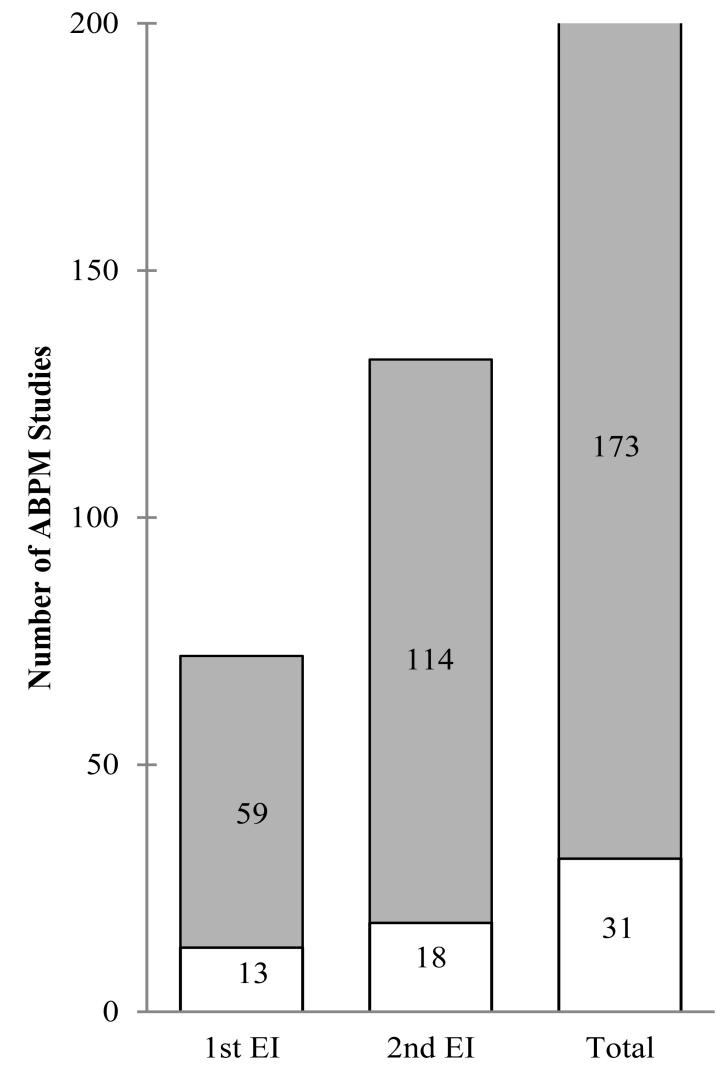

Fig. (2). Number of ABPM studies carried out following specific educational interventions. Values reflect the number of ABPM studies. 1st and 2nd EI means ABPM studies performed after the first and second specific educational interventions on ABPM, respectively. These data are summarized in total bars. Empty and filled bars correspond to ABPM records done by non-attendant and attendant professionals, respectively.

1 preferred home BP monitoring to ABPM. Three more professionals did not answer this question. One of the questions of the survey asked about reasons for ordering ABPM. Five possible answers were stated for this questions including 1) better diagnosis of EH; 2) better detection of the white coat effect; 3) evaluation of response to treatment; 4) information on circadian cycle, 5) reduction in number of consultations. All professionals from attendant HBUs checked answers 1 to 4 , while most professionals from nonattendant HBUs did not answer this question. Similarly, when we asked about the convenience of this procedure, all professionals from attendant HBUs answered this question, but the answers of nurses and doctors differed: most nurses were concerned about possible discomfort of patients while doctors were concerned with complexities in filling out the order forms. Again, most professionals from non-attendant HBUs did not answer this question.

Finally, as a third approach, we assessed whether the results of ABPM had had any influence in modifying the treatment of patients. (We should mention that there are distinct normal or optimal BP values depending on the method used to evaluate BP and these values are summarized in Table 1).

With regard to normal values for ABPM, we found a high percentage of riser patients that were badly controlled $(79 \%)$. Based upon these results, 37 re-measurements should 
have been performed, but only 10 were carried out (27\%). The attendant and non-attendant professionals performed 8 and 2 re-measurements, respectively.

Table 1. Normal and Optimal Values of Blood Pressure (BP)

\begin{tabular}{|c|c|c|}
\hline & Normal Values & Optimal Values \\
\hline \hline Office BP & $<140<90$ & $<135<85$ \\
\hline Home BP & $<135<85$ & $<130<80$ \\
\hline ABPM * & $<130<80$ & $<125<75$ \\
\hline \multicolumn{2}{|c|}{ Left and right values in cells stand for systolic and diastolic blood pressure, } \\
respectively. $\left(^{*}\right.$ ABPM, ambulatory blood pressure monitoring $[2,3,6,8,10]$.
\end{tabular}

\section{DISCUSSION}

In this study we presented data on the implementation of ABPM in a primary care center in the metropolitan area of Barcelona. Implementation was driven by two mentor nurses who carried out four educational sessions for health professionals, including nurses and family doctors. This study was carried out during a two-year follow-up period and to our knowledge this is the first study to address these issues. We assessed the outcome of attendance at the educational sessions at three different levels. First, we assessed whether these sessions increased the number of orders of ABPM. Second, we used a survey to evaluate to what extent the input of our educational sessions was understood by attendants. Third, we analyzed the effect ABPM results had on the treatment of patients with bad control of their BP.

The study involved 204 individuals similarly distributed by gender but men had a mean of age 7 years older than women. This difference is in concordance with our data on the total population affected by EH and controlled in our primary care center [18]. Clinical characteristics of the 204 analyzed individuals (including ABPM records) matched with those described in several reports published by the Spanish CARDIOSRISK/MAPAPRES Group but some points should be highlighted. First, in our group of individuals, we found a high percentage of riser patterns $(20 \%)$, 2-fold higher than that described in the MAPAPRES study (10\%) [15]. This marked difference could be due to the number of individuals included in our study $(n=204)$ compared with the number evaluated in the CARDIOSRISK/MAPAPRESSTUDY $(\mathrm{n}=38,000)$. However, these differences may also be explained by the priority given to ABPM measurements to patients with complicated $\mathrm{EH}$ or to differences in the mean of ages of the populations in the two studies. It is our opinion that the reason for the difference is revealed in the order form statement evaluation: The most frequent given for ordering an ABPM study was to know treatment efficacy and secondly, to identify a white coat syndrome or to assess whether treatment of high risk EH patients is correctly conducted. Conversely, the identification of a circadian rhythm or labile hypertension was stated in a lower number of ABPM orders suggesting that most ABPM studies were done to assess good practice or to correct a therapeutic regime. Secondly, keeping in mind that good control in ABPM is related to a more restricted normal range of systolic and diastolic BPs (see Table 1), in our series, good control was only found in 45 and $50 \%$ of patients with a dipper or non-dipper ABPM pattern, respectively. This percentage was even worse among extremely dipper patients $(40 \%)$ and risers $(29 \%)$, patterns associated to a higher risk of cardiovascular events. We assessed CVR among our set of EH individuals in order to know how BP was controled among high CVR. As depicted in Fig. (1C), 22\% $(n=45)$ had a non-added or low CVR while most of our EH patients had a moderate, high or very high CVR $(78 \%$; $n=159)$. We analyzed also how EH was controled with respect to CVR (Fig. 1D). A good control of BP was observed among 57$60 \%$ of patients with low CVR whereas was higher among patients with a low CVR patients. These data indicated that BP was better controlled in patients with a low CVR and conversely, BP was poorly controlled among patients with a higher cardiovascular risk that demands a stricter and better control. Unfortunately, this is reported in most of studies that address these issues [3, 8-12].

In our study we had the chance to compare attendants and non-attendants (control group) professionals because they were distributed in a similar number. Attendance at the educational interventions appeared to be crucial for the outcome of good practice regarding ABPM. Differences in the number of orders of ABPM per month and other responses to the survey support this. Attendants ordered 5.6fold more ABPM than non-attendants. Results from our survey show a clear difference among attendants and nonattendants with respect to their knowlegment on ABPM, BP pattern and their manegement. No scientific reasons were argued by non- attendants against ABPM. Furthermore, a better understanding of $\mathrm{EH}$ characteristics was apparent among attendant professionals. We should keep this in mind in the future and try to make a stronger effort to motivate and achieve wider participation in EH training sessions. New strategies such as keeping continuous HBU information on ABPM orders and performing new ABPM on riser patients could be applied in the future.

Once we have obtained the results of an ABPM, do we know how to manage the patient? Which therapeutic changes should be introduced based upon these results? To explore the consequences of ABPM results on therapeutic behavior we further analyzed the patient group with a riser pattern. Theoretically, therapeutic modifications should have been provided for patients in this group based upon their ABPM patterns and a second measurement should have been performed to ensure that correct control has been achieved. Thus, we checked how many re-measurements were performed in this specific group. A 5-fold and a 2.5-fold higher cardiovascular risk has been claimed for this group of patients in comparison to non-EH individuals with nonadded cardiovascular risk or to $\mathrm{EH}$ individuals with a dipping pattern, respectively [3, 8-12, 19]. In our group of 40 risers, 37 were available for a second ABPM if a therapeutic change was introduced. 28 of these individuals were seen by attendant professionals but a second ABPM was performed in only 8 patients. These figures are even worse among nonattendants: only 2 re-measurements were performed among the 9 available riser patients. In summary, a second ABPM was carried out in only 10 out of 37 risers. It should be mention that results on the type of BP pattern are indicated in the ABPM reports as well as a comment on the sequential BP measurements. For example, a report such as "good control", regarding SBP and DBP could be followed with a 
comment as"riser pattern". The latter comment might not be taken into consideration so that subjects with good BP control but with a riser pattern might not receive changes in their therapeutic regime and thus, a second ABPM was not ordered. For example, among 27 risers, although 9 were under good BP control, the riser pattern should have indicated changes in their treatment and follow-up with a second ABPM. However, a second ABPM was done in only 3 patients. These data reflect a misunderstanding of one of our messages as mentors and suggest that we need to design new strategies to resolve these issues.

\section{CONCLUSIONS}

Taken together, our study suggest that specific training processes are needed for implementation of ABPM and an even more concentrated effort should be focused on training in the correct interpretation of ABPM results. Thus, followup of these educational interventions is mandatory.

\section{CONFLICT OF INTEREST}

The authors confirm that this article content has no conflict of interest.

\section{ACKNOWLEDGEMENTS}

We thank Dr. Lindsey A. Miles for her critical revision of this manuscript. This work was supported by Fondo Investigación Sanitaria 08/0956, MJ.P-L and JF.

\section{REFERENCES}

[1] Chobanian AV. Major improvements in the control of hypertension in Canada. CMAJ 2011; 183: 996-7.

[2] Syrseloudis D, Andrikou I, Andrikou E, Dimitriadis K, Stefanadis C. Ambulatory blood pressure monitoring in resistant hypertension. Int J Hypertens 2011; 2011: 1-4.

[3] Kim SG. Clinical implications of ambulatory and home blood pressure monitoring. Korean Circ J 2010; 40: 423-31.

[4] Pater C. Beyond the Evidence of the New Hypertension Guidelines. Blood pressure measurement - is it good enough for accurate diagnosis of hypertension? Time might be in, for a paradigm shift (I). Curr Control Trials Cardiovasc Med 2005; 6: 114.
[5] Stergiou GS, Parati G. Home blood pressure monitoring may make office measurements obsolete. J Hypertens 2012; 30: 463-5.

[6] Pickering TG, Shimbo D, Haas D. Ambulatory blood-pressure monitoring. N Engl J Med 2006; 354: 2368-74.

[7] vad der Wel MC, Bunk IE, van Weel C, et al. A novel approach of office blood pressure measurement: 30 minute office blood pressure $v s$ daytime ambulatory blood pressure. Ann Fam Med 2011; 9: 128-35.

[8] Madin K, Iqbal P. Twenty four hour ambulatory blood pressure monitoring: a new tool for determining cardiovascular prognosis. Postgrad Med J 2006; 82: 548-51.

[9] Eguchi K. Ambulatory blood pressure monitoring in diabetes and obesity-a review. Int J Hypertens 2011; 2011: 954757.

[10] Eguchi K. A call for wider use of ambulatory blood pressure monitoring in patients with diabetes. Hypertens Res 2011; 34: 1171-2.

[11] O'Brien E, Sheridan J, O'Malley K. Dippers and non-dippers. Lancet 1988; 13: 397.

[12] Parati G, Bilo G. Should 24-h ambulatory blood pressure monitoring be done in every patient with diabetes? Diabetes Care 2009; 32 (Suppl 2): 298-304.

[13] Redon J, Bilo G, Parati G. Home blood pressure control is low during the critical morning hours in patients with hypertension: the SURGE observational study. Fam Pract 2012; 29 (4): 421-6.

[14] Iqbal P, Stevenson L. Cardiovascular outcomes in patients with normal and abnormal 24-hour ambulatory blood pressure monitoring. Int J Hypertens 2011; 2011: 786912.

[15] Llisterri JL, Alonso FL, Gorostidi M, et al. Proyecto CARDIORISC-MAPAPRES. Sociedad Española de Hipertensión-Liga Española para la Lucha contra la Hipertensión Arterial (SEH-LELHA). Differences between office and ambulatory control of hypertension in very elderly patients. The CARDIORISC - MAPAPRES project]. Med Clin (Barc) 2009; 28: 769-76.

[16] Stergiou GS, Parati G, Asmar R, et al. on behalf of the European Society of Hypertension Working Group on Blood Pressure Monitoring. Requirements for professional office blood pressure monitors. J Hypertens 2012; 30: 463-5.

[17] Bladé-Creixenti J, Pascual-Moron I, Gómez-Sorribes A, et al. Grupo de estudio Dirección Clínica y Enfermedad Cardiovascular. Impact on cardiovascular health results of the introduction of the clinical governance contract into primary care in Tarragona. Aten Primaria 2006; 37: 51-5.

[18] Freixenet N, Moreno-Rosel MS, Barceló MJ, et al. Detection of hereditary hemochromatosis and biochemical iron overload in primary care: a multicenter case finding study in Spain. Am J Hematol 2010; 85: 294-6.

[19] Eguchi K, Kario K. Ambulatory versus home versus clinic blood pressure. Hypertension 2012; 59(3): e25. 